\title{
Zum Schutzpotential des Asylbereichs für Menschenhandelsopfer
}

\section{Völkerrechtliche Vorgaben, Status quo und Handlungsbedarf}

\section{Nula Frei *}

Menschenhandelsopfer haben kraft Völker- und Europarecht besonderen Anspruch auf Schutz und Unterstützung. Der Beitrag stellt dar, wie diese Opferschutzverpflichtungen im Asylverfahren umgesetzt werden können: Wann erfüllen Menschenhandelsopfer die Flüchtlingseigenschaft? Wie kann sichergestellt werden, dass sie im Asylverfahren als Opfer erkannt werden? Wie sollte ein völkerrechtskonformes Identifizierungsverfahren aussehen? Wie wird die Unterstützung im Asylverfahren gewährleistet und wie verhält sich der Opferschutz zum Dublin-Verfahren? Letztlich ist festzustellen, dass der Asylbereich sein Schutzpotential im heutigen Zeitpunkt sowohl in Bezug auf die materielle Schutzgewährung als auch auf die Behandlung von Opfern im Verfahren noch nicht ganz ausschöpft.

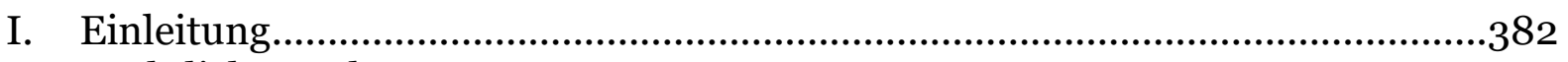

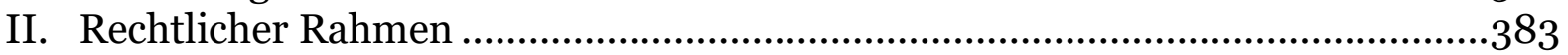

III. Möglichkeit der materiellen Schutzgewährung …...........................................385

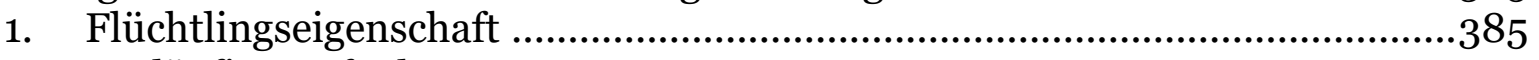

2. Vorläufige Aufnahme ..................................................................... 387

3. Schwierigkeiten der Glaubhaftigkeitsbeurteilung bei

Menschenhandelsopfern ..................................................................... 387

IV. Opferschutz als Prozess: Umsetzung im Asylverfahren ............................... 388

1. Erkennung und Anschlussverpflichtungen ......................................... 388

2. Formelle Identifizierung ............................................................... 390

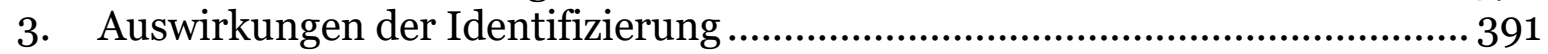

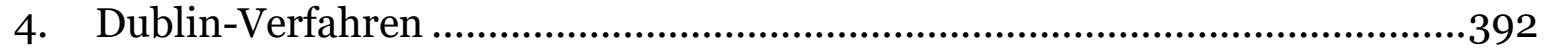

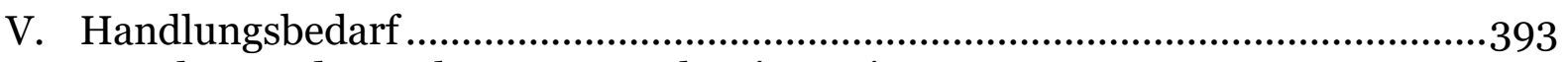

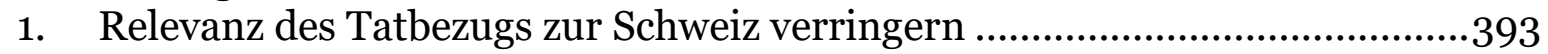

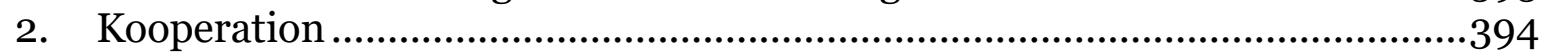

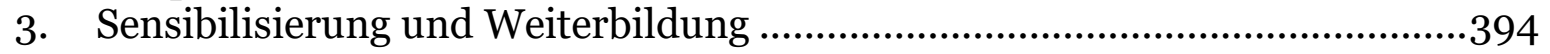

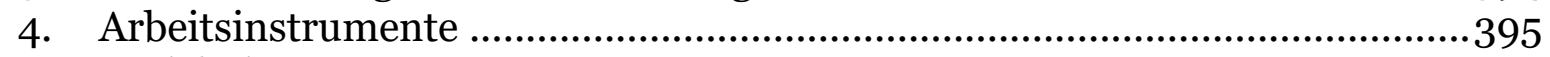

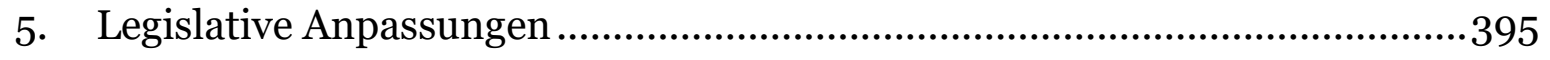

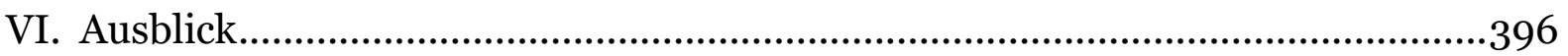

Zitiervorschlag: Nula Frei, Zum Schutzpotential des Asylbereichs für Menschenhandelsopfer, in: sui-generis 2018, S. 381

URL: $\quad$ sui-generis.ch/82

DOI: $\quad$ https://doi.org/10.21257/sg.82

* Dr. iur. Nula Frei (nula.frei[at]unifr.ch) ist Oberassistentin am Institut für Europarecht der Universität Fribourg.

Dieses Werk ist lizenziert unter einer Creative Commons Namensnennung - Weitergabe unter gleichen Bedingungen 4.0 International Lizenz. 


\section{Einleitung}

1 Die junge Frau aus Nigeria landete am Flughafen Genf und stellte umgehend einen Asylantrag. Nach den ersten üblichen Abklärungen durch die Behörden wurde sie aufgrund ihres Alters einer Asylunterkunft für Minderjährige im Kanton Genf zugeteilt und erhielt ein Zugbillet, um dorthin zu reisen. In der Asylunterkunft kam sie jedoch nie an. Ein halbes Jahr später wurde sie von der französischen Polizei bei einer Razzia in einem Bordell angehalten und in Haft genommen. Aufgrund ihrer im Asylverfahren abgegebenen Fingerabdrücke stellten die französischen Behörden einen Dublin-Wiederaufnahmeantrag an die Schweiz.

Solche und andere Fälle, in denen ein Verdacht auf Menschenhandel ${ }^{1}$ besteht, wurden in den letzten Jahren im Asylverfahren immer häufiger entdeckt. Verschiedene Konstellationen können dabei unterschieden werden. Nebst Fällen wie

\footnotetext{
Die völkerrechtlich normierte und allgemein anerkannte Definition von Menschenhandel findet sich im sog. Palermo-Protokoll der Vereinten Nationen (Zusatzprotokoll vom 15. November 2000 zur Verhütung, Bekämpfung und Bestrafung des Menschenhandels, insbesondere des Frauen- und Kinderhandels zum Übereinkommen der Vereinten Nationen gegen die grenzüberschreitende organisierte Kriminalität, SR 0.311.542). Spätere Abkommen, insbesondere auch die Europaratskonvention zur Bekämpfung des Menschenhandels (Konvention des Europarats vom 16. Mai 2005 zur Bekämpfung des Menschenhandels, EMK; SR 0.311.543), haben diese Definition wörtlich übernommen und auch der EGMR verwendet sie zur Auslegung von Art. 4 EMRK (Konvention vom 4. November 1950 zum Schutze der Menschenrechte und Grundfreiheiten, SR 0.101). Gemäss Art. 3 lit. a des Palermo-Protokolls ist Menschenhandel «die Anwerbung, Beförderung, Verbringung, Beherbergung oder Aufnahme von Personen durch die Androhung oder Anwendung von Gewalt oder anderen Formen der Nötigung, durch Entführung, Betrug, Täuschung, Missbrauch von Macht oder Ausnutzung besonderer
}

dem eingangs geschilderten, bei denen der Verdacht besteht, dass die Asylgesuchstellung lediglich eine Strategie der Täter darstellte, um das Opfer in den Schengen-Raum zu «schleusen», kommt es auch vor, dass Personen, die in einem anderen europäischen Staat ausgebeutet wurden, in der Schweiz Schutz suchen oder dass Personen als Grund für die Asylgesuchstellung geltend machen, ihnen würde bei einer Rückkehr in ihr Herkunftsland Menschenhandel drohen.

3 Zwischen Anfang 2014 und Ende 2017 wurden über 280 Personen im Asylverfahren durch das Staatssekretariat für Migration (SEM) als potentielle Opfer von Menschenhandel eingeschätzt; davon stammte der überwiegende Teil aus Nigeria, gefolgt von Eritrea, Äthiopien, Kongo und Kamerun als Herkunftsländern. Über 80 \% der Verdachtsfälle betreffen Frauen; rund $12 \%$ Minderjährige. ${ }^{2}$

4 Der migrationsrechtliche Umgang mit Menschenhandelsopfern wurde in der Schweiz seit dem Aufkommen des Themas in den späten Neunzigerjahren hauptsächlich in Bezug auf das durch die Kantone vollzogene Ausländerrecht diskutiert. Der Asylbereich ist hingegen sowohl in der Praxis wie auch in der Wissenschaft unterrepräsentiert und kommt erst allmählich in den Fokus. Dementsprechend findet sich auch noch immer

Hilflosigkeit oder durch Gewährung oder Entgegennahme von Zahlungen oder Vorteilen zur Erlangung des Einverständnisses einer Person, die Gewalt über eine andere Person hat, zum Zweck der Ausbeutung. Ausbeutung umfasst mindestens die Ausnutzung der Prostitution anderer oder andere Formen sexueller Ausbeutung, Zwangsarbeit oder Zwangsdienstbarkeit, Sklaverei oder sklavereiähnliche Praktiken, Leibeigenschaft oder die Entnahme von Organen.»

2 Quelle: Statistikdienst des SEM, Statistiken der Autorin vorliegend. 
häufig die Vorstellung, dass Menschenhandel keine Asylrelevanz hat, sondern ein rein strafrechtliches Problem darstellt. Das sieht man auch an der «Schutzquote» im Asylbereich, die zudem noch nach Herkunftsland divergiert, so wurde etwa bei den 91 Personen aus Nigeria in keinem einzigen Fall Asyl gewährt, acht Frauen aus Nigeria wurden vorläufig aufgenommen, zehn wurden nach Nigeria weggewiesen und bei 30 Personen erging aufgrund der Zuständigkeit eines anderen Staates ein DublinNichteintretensentscheid. Dissertation, die nun publiziert wurde3, befasst sich mit diesem Themenkomplex und analysiert die Umsetzung der völkerrechtlichen Vorgaben zum Umgang mit Menschenhandelsopfern im schweizerischen Asylverfahren. Vorliegend werden die wichtigsten Ergebnisse dieser Publikation dargestellt. In einem ersten Schritt werden die anwendbaren rechtlichen Standards dargestellt, wobei sich zeigt, dass eine sehr breite und mittlerweile sehr ausdifferenzierte rechtliche Basis besteht (II.). Ein zweiter Teil behandelt die materiellen Fragen der Schutzgewährung im Asylverfahren: Unter welcher Voraussetzung erfüllen Menschenhandelsopfer die Flüchtlingseigenschaft, wann liegen Wegweisungsvollzugshindernisse vor, die zu einer vorläufigen Aufnahme führen? (III.). Der dritte Teil schliesslich untersucht, wie die

Nula Frei, Menschenhandel und Asyl. Die Umsetzung der völkerrechtlichen Vorgaben zum Opferschutz im Schweizerischen Asylverfahren. Nomos/Stämpfli, 2018. Die Arbeit ist in der Nomos e-Library elektronisch verfügbar; ab Januar 2020 auch open access. Einige Absätze im vorliegenden Beitrag wurden wörtlich aus dieser Arbeit übernommen.
Schutzverpflichtungen zugunsten von Menschenhandelsopfern ins Asylverfahren - also in prozessualer Hinsicht - einzugliedern sind (IV.). Entsprechend der bisher noch nicht weit fortgeschrittenen Diskussion in der Praxis um die Umsetzung dieser Verpflichtungen im Asylverfahren stellt die Arbeit an vielen Stellen Handlungsbedarf fest (V.).

\section{Rechtlicher Rahmen}

6 Für den Umgang mit Menschenhandelsopfern besteht mittlerweile eine sehr breite völkerrechtliche wie auch eine solide verfassungsrechtliche Basis, die sich teilweise auch immer noch stark weiterentwickelt. Dabei kann unterschieden werden zwischen «allgemeinen» Grundlagen, die sämtliche Menschen vor Menschenhandel schützen, so insbesondere das sog. Palermo-Protokoll der Vereinten Nationen sowie das - etwas differenziertere - Übereinkommen des Europarats zur Bekämpfung des Menschenhandels, welche spezifische Pflichten der Staaten in den Bereichen Prävention, Strafverfolgung, Kooperation sowie Opferschutz aufstellen. Auch die allgemeinen menschenrechtlichen Sklavereiverbote in Art. 8 UNO-Pakt II4 sowie Art. 4 EMRK sind auf Menschenhandelssachverhalte anwendbar; und insbesondere der Europäische Gerichtshof für Menschenrechte (EGMR) übernimmt je länger, je mehr die Garantien aus dem Europaratsübereinkommen zur Bekämpfung des Menschenhandels als positive Schutzpflichten der Staaten bei Fällen von Menschenhandel gemäss Art. 4

4 Internationaler Pakt vom 16. Dezember 1966 über bürgerliche und politische Rechte, (UNOPakt II; SR 0.103.2). 
EMRK.5 Der Schutz vor Menschenhandel und Ausbeutung ist in der Schweiz auch verfassungsrechtlich verankert, namentlich als Teilgehalt der persönlichen Freiheit (Art. 10 Abs. 2 BV6) sowie der Wirtschaftsfreiheit (Art. 27 BV).7 Darüber hinaus enthalten zahlreiche spezifische Abkommen einen verstärkten Schutz für bestimmte als besonders schutzbedürftig geltende Gruppen, namentlich Frauen und Mädchen ${ }^{8}$, Kinder ${ }^{9}$, sowie Menschen mit einer Behinderung ${ }^{10}$, der sich im schweizerischen Verfassungsrecht in den Diskriminierungsverboten zugunsten von Frauen (Art. 8 Abs. 3 BV) und Menschen mit einer Behinderung (Art. 8 Abs. 4 BV) sowie dem Schutz von Kindern und Jugendlichen (Art. 11 BV) spiegelt.

Vgl. Nula Frei, Identifizieren, Schützen, Unterstützen: Neue Rechtsprechung des EGMR zum Opferschutz bei Menschenhandel, in: ASYL 3/2017, S. 21.

6 Bundesverfassung der Schweizerischen Eidgenossenschaft vom 18. April 1999 (BV; SR 101).

Frei, Menschenhandel und Asyl (Fn. 3), S. 112 ff.

8 So insb. Art. 6 des Übereinkommens vom 18. Dezember 1979 zur Beseitigung jeder Form von Diskriminierung der Frau (CEDAW; SR o.108) sowie die jüngst auch von der Schweiz ratifizierte Istanbul-Konvention (Übereinkommen des Europarats vom 11. Mai 2011 zur Verhütung und Bekämpfung von Gewalt gegen Frauen und häuslicher Gewalt; SR 0.311.35).

9 Übereinkommen der ILO Nr. 182 vom 17. Juni 1999 über das Verbot und unverzügliche Massnahmen zur Beseitigung der schlimmsten Formen der Kinderarbeit (SR 0.822.728.2); Art. 3236 des Übereinkommens vom 20. November 1989 über die Rechte des Kindes (Kinderrechtskonvention; SR 0.107); Fakultativprotokoll vom 25. Mai 2000 zum Übereinkommen über die Rechte des Kindes betreffend den Verkauf von Kindern, die Kinderprostitution und die Kinderpornographie (SR 0.107.2); sowie das Übereinkommen des Europarats vom 25. Oktober 2007 zum Schutz von Kindern vor sexueller Ausbeutung und sexuellem Missbrauch, (LanzaroteKonvention; SR 0.311.40).

10 Art. 16 und 27 des Übereinkommens vom 13. Dezember 2006 über die Rechte von Menschen mit Behinderungen (Behindertenrechtskonvention; SR 0.109).
7 Aus dem Zusammenspiel dieser Normen ergeben sich sehr detaillierte und weitreichende staatliche Verpflichtungen des Opferschutzes, welche unabhängig von der Durchführung eines Strafverfahrens gewährleistet werden müssen. ${ }^{11}$ Die Verpflichtungen decken das gesamte Spektrum vom Erstkontakt mit dem potentiellen Opfer bis zur Integration oder zur Rückkehr in den Heimatstaat ab: Staaten müssen Massnahmen treffen, um die Erkennung von Opfern durch sämtliche Stellen sicherzustellen, die in Kontakt mit Opfern kommen können. Sobald eine Person als potentielles Opfer erkannt wird, geniesst sie Schutz vor Ausweisung bis zur definitiven Identifizierung als Opfer, sie hat Anspruch auf eine Erholungs- und Bedenkzeit, während der sie einen Entscheid über ihre Kooperation mit den Behörden fällen kann, sie hat Anrecht auf Schutz ihrer physischen Integrität (etwa vor Behelligungsversuchen der Täterschaft) und sie muss Unterstützung finanzieller oder anderer Art erhalten, um ihren Lebensunterhalt sicherzustellen und ihre Rehabilitation zu ermöglichen. In begründeten Fällen hat die Person Anspruch auf die Ausstellung einer Aufenthaltsbewilligung. Sofern sie in ihren Heimatstaat zurückkehrt (wobei diese Entscheidung, wenn immer möglich, freiwillig sein soll), muss der Staat sicherstellen, dass diese Rückkehr in Sicherheit und Würde erfolgt und kein re-trafficking erfolgt. Zudem ist das Verbot des Refoulement zu beachten.

8 Diese positiven Pflichten der Staaten, die - mit Ausnahme der Erteilung eines Aufenthaltstitels und der Ermöglichung einer sicheren Rückkehr - bereits im «Verdachtsstadium», d.h. noch vor einer endgültigen Identifizierung als Opfer,

11 Frei, Menschenhandel und Asyl (Fn. 3), S. 155 ff. 
gewährleistet werden müssen, sind sowohl bei der materiellen Schutzgewährung im Rahmen der Auslegung des Flüchtlingsbegriffs und der Zulässigkeit des Wegweisungsvollzugs zu beachten (dazu sogleich, III.), wie auch in prozeduraler Weise in das Asylverfahren einzugliedern (unten, IV.).

\section{Möglichkeit der materiellen Schutzgewährung}

9 Viele Opfer sind im Fall einer Rückkehr in ihren Heimatstaat in Gefahr, Menschenrechtsverletzungen zu erleiden, beispielsweise durch erneuten Menschenhandel (sog. re-trafficking), Vergeltungsmassnahmen oder sozialen Ausschluss und Stigmatisierung. Die völkerrechtlichen Vorgaben verpflichten die Staaten dazu, Voraussetzungen zu schaffen, um den Aufenthalt der Opfer zu regularisieren, entweder aufgrund ihrer persönlichen Situation oder für ihre Zusammenarbeit mit den Strafverfolgungsbehörden. ${ }^{12}$ Im Asylverfahren wird geprüft, ob eine Person die Flüchtlingseigenschaft erfüllt und folglich Asyl erhält (1.) oder ob sie, falls das Asylgesuch abgewiesen wird, aufgrund eines Wegweisungsvollzugshindernisses vorläufig aufgenommen werden muss (2.). Dabei sind in der Praxis verschiedene Schwierigkeiten bei der Glaubhaftigkeitsbeurteilung zu beobachten (3.).

\section{Flüchtlingseigenschaft}

10 Die Flüchtlingseigenschaft besteht gemäss Art. 1 A Abs. 2 GFK ${ }^{13}$ und Art. 3 AsylG ${ }^{14}$ aus den kumulativ zu erfüllenden Elementen Verfolgung, Abwesenheit staatlichen Schutzes, Anknüpfung an ein Konventionsmerkmal sowie Begründetheit der Furcht.

11 Bei Opfern von Menschenhandel können sich insbesondere drei Verfolgungskonstellationen stellen: «re-trafficking», Vergeltungs- und Einschüchterungsmassnahmen sowie Diskriminierung und sozialer Ausschluss. Während alle drei Konstellationen in der Regel die Intensitätserfordernisse für eine flüchtlingsrelevante Verfolgung erfüllen, ist festzustellen, dass in der schweizerischen Praxis vor allem Vergeltungsmassnahmen sowie Ausbeutungsformen, die unter das Verbot der unmenschlichen oder erniedrigenden Behandlung fallen, als Verfolgung anerkannt worden sind.15 Menschenhandel im Sinne der Definition des Palermo-Protokolls wurde soweit ersichtlich noch nie explizit als Verfolgung qualifiziert.

12 Staatlicher Schutz ist bei Menschenhandel häufig in Bezug auf private Verfolgung zu prüfen, da es in der Regel nicht der Heimatstaat ist, der den Menschenhandel begeht (ihn allenfalls aber toleriert), sondern private Gruppen oder Einzelpersonen. In diesem Fall muss geprüft werden, ob der Staat genügenden

$13 \overline{\text { Abkommen vom 28. Juli } 1951 \text { über die Rechts- }}$ stellung der Flüchtlinge (SR 0.142.30).

14 Asylgesetz vom 26. Juni 1998 (SR 142.31).

15 Urteile des Bundesverwaltungsgericht D-5828/ 2010 vom 29. August 2012, E. 8.1; E-4864/2006 vom 29. Januar 2009, E. 3.4; D-262/2017 vom 1. Mai 2017, E. 5.2 sowie D-7552/2010 vom 28. Oktober 2010. 
Schutz vor dieser privaten Verfolgung bietet. Zur Einschätzung der staatlichen Schutzfähigkeit können insbesondere die völker- und menschenrechtlichen Schutzverpflichtungen gegenüber Menschenhandelsopfern, die im ersten Teil erarbeitet wurden, hilfreich sein: Wenn ein Staat diese gewährleistet, besteht eine Vermutung, dass er genügenden Schutz vor Menschenhandel bietet. In der Schweizer Gerichtspraxis wird allerdings das Vorliegen staatlichen Schutzes häufig nur rudimentär geprüft und es wird auf das grundsätzliche Vorhandensein von staatlichen Schutzstrukturen (beispielsweise eine funktionierende Polizei) verwiesen, ohne zu prüfen, ob diese im individuellen Fall für ein Menschenhandelsopfer vorhanden sind. ${ }^{16}$

Was das Anknüpfen des Menschenhandels an eines der fünf Konventionsmerkmale betrifft, wird das Merkmal «Zugehörigkeit zu einer bestimmten sozialen Gruppe» am häufigsten diskutiert. Dabei lassen sich bei Menschenhandelsopfern verschiedene soziale Gruppen identifizieren, die insbesondere durch gemeinsame Vulnerabilitätsmerkmale charakterisiert sind (etwa junge Frauen, ethnische Minderheiten, sozial benachteiligte Kinder), und deren Zugehörigkeit (auch) ursächlich für den Menschenhandel sein kann. Drohen bei einer Rückkehr ins Heimatland Vergeltungsmassnahmen oder sozialer Ausschluss, so ist es die $\mathrm{Zu}$ gehörigkeit der ehemaligen Menschenhandelsopfer zur bestimmten sozialen Gruppe, die kausal für die Verfolgung

16 Urteile des Bundesverwaltungsgericht E-3826/ 2006 vom 25. Januar 2010, E. 4.3; D-2695/2011 vom 27. Mai 2011; E-3110/2013 vom 12. Juni 2013, E. 5.1; D-1683/2014 vom 12. August 2014, E. 6.2; D-3761/2006 vom 11. September 2007, E. 5.2. ist. ${ }^{17}$ In der schweizerischen Praxis wurde dies allerdings noch in keinem Fall von Menschenhandel anerkannt. Zudem wird die Kausalität des Konventionsmerkmals für die (drohende) Verfolgung in der Praxis häufig implizit verneint, indem Menschenhandel (unzutreffend) als eine «gemeinrechtliche Straftat» mit rein gewinnsüchtigen Motiven bezeichnet wird $^{18}$, ohne anzuerkennen, dass Menschenhandel mitnichten ein «neutrales» Verbrechen ist, sondern sich praktisch immer gegen (diskriminierungsrechtlich geschützte) besonders benachteiligte Gruppen richtet.

14 Die Analyse der Rechtsprechung des Bundesverwaltungsgerichts lässt erkennen, dass die Beurteilung der Flüchtlingseigenschaft von Menschenhandelsopfern in der schweizerischen Praxis sehr restriktiv ist und hinter den international geführten Diskussionen zurückbleibt, was dazu führt, dass nur in äusserst seltenen Fällen bei Menschenhandelsopfern Asyl gewährt wird. Zudem liegt der Beurteilung häufig ein stereotypes Bild davon zugrunde, wie Menschenhandel abläuft resp. wie sich ein Menschenhandelsopfer (angeblich) verhält; dies wirkt sich auf die materielle Beurteilung der Flüchtlingseigenschaft aus und führt auch dazu, dass die Aussagen von Menschenhandelsopfern häufig als unglaubhaft qualifiziert werden (dazu unten, 3.).

$17 \overline{\text { Frei, Menschenhandel und Asyl (Fn. 3), S. } 276 \mathrm{ff}}$. m.w.H.

18 Siehe etwa jüngst Urteil des Bundesverwaltungsgericht D-2759/2018 vom 2. Juli 2018, S. 6: «dass Zwangsprostitution somit im Allgemeinen nicht an ein flüchtlingsrechtliches Merkmal anknüpft, sondern es sich dabei um ein kriminell motiviertes Verbrechen handelt», mit Verweis auf weitere Urteile. 


\section{Vorläufige Aufnahme}

15 Wird die Flüchtlingseigenschaft verneint oder liegen Asylausschlussgründe vor, muss - als Ausfluss des Refoulementverbots sowie in Beachtung humanitärer Erwägungen - das Vorliegen allfälliger Wegweisungsvollzugshindernisse geprüft werden.

Eine Unzulässigkeit des Wegweisungsvollzugs (also die Verletzung völkerrechtlicher Refoulementverbote, Art. 83 Abs. 3 AuG'19) kann bei Menschenhandelsopfern auf einer Verletzung des Verbots der Folter, unmenschlichen oder erniedrigenden Behandlung ${ }^{20}$ beruhen, da Menschenhandel, re-trafficking, Vergeltungsoder Einschüchterungsmassnahmen sowie auch - in gravierenden Fällen - gesundheitliche Probleme unter dieses Verbot fallen können. ${ }^{21}$

Eine Verletzung des Verbots der Sklaverei, Leibeigenschaft und Zwangsarbeit ${ }^{22}$ ist bei menschenhandelsbezogenen Gefahren ebenfalls als Unzulässigkeitsgrund möglich. Hierfür bietet die internationale Judikatur allerdings noch keine Orientierungshilfe: Für die Beurteilung der Frage, ob staatlicher Schutz vorhanden ist, hat sich der EGMR bisher nur auf oberflächliche Kriterien verlassen, die kaum verallgemeinerbar sind; zudem handelte es sich dabei stets nur um Zulässigkeitsentscheide. ${ }^{23}$ Für diese

19 Bundesgesetz über die Ausländerinnen und Ausländer vom 16. Dezember 2005 (AuG; SR 142.20)

20 Art. 25 Abs. 3 BV, Art. 3 EMRK, Art. 7 UNO-Pakt II, Art. 3 UNO-Antifolterkonvention.

21 Frei, Menschenhandel und Asyl (Fn. 3), S. 328 ff. Im Fall Osayi Omo-Amenaghawon gegen Dänemark (2288/2013, 15. September 2015) hat der Menschenrechtsausschuss ebenfalls diese Ansicht vertreten.

22 Art. 4 EMRK; Art. 8 UNO-Pakt II.

23 Siehe insb. Urteile des EGMR 7196/10 vom
Prüfung müssten m.E. die gleichen Kriterien verwendet werden wie für die Prüfung des staatlichen Schutzes bei der Flüchtlingseigenschaft (oben, 1.). In der Praxis der Schweiz gibt es soweit ersichtlich bislang keine Fälle, in denen ein (potentielles) Menschenhandelsopfer wegen Unzulässigkeit des Wegweisungsvollzugs vorläufig aufgenommen wurde.

18 Hingegen wird in der Praxis häufig die Unzumutbarkeit (Art. 83 Abs. 4 AuG) des Wegweisungsvollzugs von Menschenhandelsopfern angenommen. ${ }^{24}$ In Frage kommen insbesondere eine medizinische Notlage sowie eine Kombination von Gründen; dabei handelt es sich in der Regel um eine stark einzelfallabhängige Entscheidung. Ebenfalls als Unzumutbarkeitskriterium in Frage kommt eine Verletzung des Kindeswohls (Art. 3 Kinderrechtskonvention).

\section{Schwierigkeiten der} Glaubhaftigkeitsbeurteilung bei Menschenhandelsopfern

19 Die Analyse der Rechtsprechung bringt ebenfalls zu Tage, dass die Glaubhaftigkeit der Aussagen von Menschenhandelsopfern oft angezweifelt oder verneint wird und das Asylgesuch deshalb basierend auf Art. 7 AsylG abgewiesen wird. Grund dafür ist meist, dass die Opfer widersprüchliche oder unwahre Aussagen machen oder sich nicht «der allgemeinen Lebenserfahrung entsprechend» verhalten, etwa indem sie nach einer Flucht wieder zu den Tätern zurückgekehrt sind oder trotz objektiv bestehender Flucht-

29. November 2011 (V.F. gegen Frankreich) und 4455/14 vom 26. Mai 2015 (L.O. gegen Frankreich) sowie die Diskussion in Frei, Menschenhandel und Asyl (Fn. 3), S. 337 ff.

24 Vgl. die Hinweise bei Frei, Menschenhandel und Asyl (Fn. 3), S. 339 ff. 
möglichkeit in der Ausbeutungssituation ausgeharrt haben. Es drängt sich der Verdacht auf, dass bei den Asylbehörden teilweise immer noch unrealistische Vorstellungen darüber vorherrschen, wie sich ein Menschenhandelsopfer typischerweise verhält. Verkannt wird dabei, dass die extremen Erfahrungen des Menschenhandels zu irrationalem Verhalten führen können, welches häufig eher für als gegen die Glaubhaftigkeit der Aussagen dieser Personen spricht. Die entsprechende Sensibilität bei den Asylbehörden muss erst noch gebildet werden (siehe auch unten, V.3.).

\section{Opferschutz als Prozess:}

\section{Umsetzung im Asylverfahren}

20

Nebst der Frage der materiellen Schutzgewährung ist in Bezug auf das Asylverfahren auch zu prüfen, welche notwendigen und möglichen Auswirkungen die Umsetzung des Menschenhandelsbekämpfungsrechts auf das Asylverfahren in der Schweiz hat. Die aktuelle Praxis enthält nur Vorgaben zum Erkennen und lässt die formelle Identifizierung weitgehend ausser Acht. ${ }^{25}$ Um die eingangs erwähnten positiven Verpflichtungen auf das Asylverfahren anzuwenden, ist es hilfreich, den Opferschutz selber als Prozess zu verstehen: Ab dem Moment des Erkennens von Verdachtsmomenten im Sinne konkreter Anhaltspunkte auf Menschenhandel greifen bereits die meisten Gewährleistungspflichten, so etwa die Gewährung einer Erholungs- und Bedenkzeit, der physische Schutz sowie die Unterstützung. Angesichts der Tragweite dieser Verpflichtungen muss m.E. das Asylverfahren ab dem Erkennen konkreter Anhaltspunkte temporär ausgesetzt

25 Frei, Menschenhandel und Asyl (Fn. 3), S. 370. werden und es müssen funktionierende Weiterverweisungsmechanismen zwischen den beteiligten Behörden und Unterstützungsorganisationen eingerichtet sein. Das Erkennen löst auch die Verpflichtung aus, ein formelles Identifizierungsverfahren durchzuführen, nach dessen Abschluss unter Umständen weitere Verpflichtungen zugunsten identifizierter Opfer eintreten.

\section{Erkennung und}

\section{Anschlussverpflichtungen}

21 Es stellt sich zunächst die Frage, wie eine völkerrechtskonforme Erkennungsstufe im Asylbereich aussehen könnte, so dass die Erkennung nicht alleine dem «selfreporting» der Opfer oder dem «Bauchgefühl» engagierter Mitarbeitender überlassen bleibt. Die Dissertation kommt zum Schluss, dass dafür ein Screening anlässlich der Erstbefragung in der Vorbereitungsphase notwendig wäre. Die zusätzlich gebotene Sensibilisierung sämtlicher «front-end» Mitarbeitenden wird aktuell bereits punktuell durchgeführt, jedoch müssten diese Bemühungen intensiviert und flächendeckend für alle Mitarbeitenden im Asylbereich umgesetzt werden, die Kontakt mit Menschenhandelsopfern haben könnten. Mandatiert das SEM Leistungserbringer für bestimmte Bereiche des Asylverfahrens, sollten Sensibilisierungsmassnahmen auch vertraglich vorgeschrieben werden. ${ }^{26}$

22 Auch den durch das Erkennen ausgelösten Anschlussverpflichtungen wie Unterstützung, Gewährung einer Erholungsund Bedenkzeit sowie physischer Schutz wird in der aktuellen Asylpraxis häufig

26 Frei, Menschenhandel und Asyl (Fn. 3), S. 383. 
noch nicht genug Rechnung getragen: Während die allgemein im Asylverfahren vorhandenen Unterstützungsleistungen der Asylsozialhilfe nicht spezifisch auf Menschenhandelsopfer zugeschnitten sind (und auch nicht als Reaktion auf das Erkennen geleistet werden, sondern zu den Grundleistungen der Asylsozialhilfe gehören), sind die existierenden spezialisierten Opferhilfeleistungen für Menschenhandelsopfer rechtlich und praktisch abhängig von einem Tatbezug zur Schweiz, weil sie derzeit nur im Rahmen der gesetzlichen Opferhilfe gewährleistet werden, die im Wesentlichen nur auf Opfer von in der Schweiz begangenen Straftaten anwendbar ist (Art. $3 \mathrm{OHG}^{27}$ ). Eine Erholungs- und Bedenkzeit, die in der Schweiz in der Verordnung zum AuG (Art. $35 \mathrm{VZAE}^{28}$ ) geregelt ist, ist aufgrund der Nichtanwendbarkeit des Ausländerrechts im Asylbereich derzeit ausgeschlossen. Ein Kantonswechsel (bspw. aus Sicherheitsgründen oder weil im aktuellen Kanton kein spezialisiertes Opferhilfeangebot besteht) scheitert in der Praxis häufig an den hohen Anforderungen, die an die Notwendigkeit eines Kantonswechsels gestellt werden, da Beschwerden gegen Zuweisungsentscheide gemäss Art. 27 Abs. 3 AsylG nur mit dem Grundsatz der Einheit der Familie begründet werden können. Es bestehen somit rechtliche wie auch praktische Hindernisse für einen völkerrechtskonformen Umgang mit Menschenhandelsopfern im Asylverfahren.

$27 \overline{\text { Bundesgesetz vom 23. März } 2007 \text { über die Hilfe }}$ an Opfer von Straftaten (OHG; SR 312.5).

28 Verordnung vom 24. Oktober 2007 über Zulassung, Aufenthalt und Erwerbstätigkeit (VZAE; SR 142.201).
23 Die für solche Massnahmen zudem notwendige Koordination zwischen verschiedenen Stellen (u.a. das SEM, die kantonal für die Unterbringung zuständigen Behörden sowie die spezialisierten Opferberatungsstellen) ist bisher praktisch nicht gewährleistet und es fehlt an definierten Abläufen. Für eine konsequente Umsetzung der völkerrechtlichen Verpflichtungen müsste ein nationaler Weiterverweisungsmechanismus speziell für den Asylbereich geschaffen werden, in welchem sämtliche relevanten Behörden sowie Nichtregierungsorganisationen zusammengeschlossen sind und Abläufe zur Weiterverweisung von (potentiellen) Opfern definiert werden. Derzeit existieren derartige Kooperationen im Asylbereich nur punktuell und ad hoc, anders als im Ausländerbereich, wo die meisten Kantone mittlerweile über «runde Tische» und geregelte Ablaufschemata verfügen.29 Ein solcher Mechanismus wäre auch schon deshalb eine geeignete Verbesserungsmassnahme, weil er im Gegensatz zu vielen anderen der hier diskutierten völkerrechtlich angezeigten Umsetzungsvorschläge ohne legislative Änderungen rasch umsetzbar wäre. Ebenfalls im Rahmen dieses Mechanismus geklärt werden müsste die Frage der Finanzierung der Opferhilfe bei Opfern mit ausländischem Tatort.

24 In prozeduraler Hinsicht hat das Erkennen konkreter Anhaltspunkte dafür, dass eine Person Menschenhandelsopfer sein könnte, insbesondere die Auswirkung, dass das Asyl- oder Wegweisungsverfahren zu suspendieren resp. ein DublinVerfahren zu beenden ist, bis das formel-

$29 \overline{\text { Siehe, anstelle vieler, der Kooperationsmecha- }}$ nismus des Kantons Freiburg gegen Menschenhandel. 
le Identifizierungsverfahren abgeschlossen ist. $3^{0}$

\section{Formelle Identifizierung}

25 Aus Art. 10 des Europaratsübereinkommens zur Bekämpfung des Menschenhandels ${ }^{31}$ sowie der Rechtsprechung des EGMR zu Art. 4 EMRK ${ }^{2}$ ergibt sich eine Pflicht, ein formalisiertes Identifizierungsverfahren für potentielle Opfer von Menschenhandel einzurichten.33 Aktuell gibt es in der Schweiz kein rechtlich vorgesehenes, einheitliches Verfahren, um ein Menschenhandelsopfer formell zu identifizieren. Vielmehr schätzen die einzelnen Fachbehörden jeweils für ihre Zwecke ein, ob es sich bei einer Person um ein Opfer von Menschenhandel handelt. Dieses fragmentierte System informeller Identifizierungen reicht nicht aus, um den völkerrechtlich vorgesehenen Schutz ausreichend zu gewährleisten, u.a. weil deren Effizienz und Wirksamkeit fraglich sind und keine Verfahrensrechte für die Opfer bestehen. Ausgehend von dieser Feststellung wurde in der Dissertation basierend auf den völkerrechtlichen Vorgaben sowie den Beispielen anderer Länder und bereits existierender multidisziplinärer Gremien in der Schweiz ein Vorschlag für einen Mechanismus entwickelt.

30 Frei, Menschenhandel und Asyl (Fn. 3), S. 440 f.

31 GRETA, das für die Überwachung der Europaratskonvention gegen Menschenhandel zuständige Expertengremium, empfiehlt den Staaten in konstanter Praxis, einen formalisierten Identifizierungsmechanismus einzurichten, vgl. ansteller vieler: GRETA, Report concerning the implementation of the Council of Europe Convention on Action against Trafficking in Human Beings by Switzerland, GRETA(2015)18, 14. Oktober 2015, Ziff. 129.

32 Insb. Urteil des EGMR 71545/12 vom 21. Januar 2016 (L.E. gegen Griechenland).

33 Frei, Menschenhandel und Asyl (Fn. 3), S. 160 ff.
26 Vorgeschlagen wird die Einrichtung einer Identifizierungskommission, die bei der Koordinationsstelle gegen Menschenhandel und Menschenschmuggel (KSMM) angegliedert sein könnte und sich aus verschiedenen staatlichen und nichtstaatlichen Akteuren in der Schweiz zusammensetzt, die eine Rolle bei Erkennung, Schutz und Unterstützung der Opfer spielen.34 Diese Kommission müsste eine eigene Geschäftsstelle haben, welche Meldungen über Verdachtsfälle entgegennimmt und untersucht, die von definierten Erstkontaktstellen wie dem SEM, der Rechtsvertretung sowie den Opferberatungsstellen an die Kommission gelangen. Die Geschäftsstelle könnte die Unterlagen für den formellen Identifizierungsentscheid vorbereiten und sie der Kommission zur Entscheidung vorlegen. Gegen die Identifizierungsentscheide der Kommission muss aus verfassungs- und menschenrechtlicher Sicht eine Beschwerdemöglichkeit bei einer unabhängigen Instanz, beispielsweise beim Bundesverwaltungsgericht, bestehen.

Die Umsetzung eines solchen Modells würde kurzfristig einen nicht unerheblichen Koordinations-, Planungs-, Anschubs- und Ressourcenaufwand verursachen. Insgesamt würde das Modell aber längerfristig zu Einsparungen führen, die Qualität der Identifizierungsentscheide massgeblich verbessern, eine transparente und faire Entscheidfindung ermöglichen und die notwendigen Verfahrensrechte für die Opfer gewährleisten. In anderen Staaten, etwa im Vereinigten Königreich, werden ähnliche Modelle bereits seit mehreren Jahren prak-

$34 \overline{\text { Frei, Menschenhandel und Asyl (Fn. 3), S. } 444 \mathrm{ff} .}$ 
tiziert.35 Quasi als ein «Nebenprodukt» würde mit der Identifizierungskommission zudem eine Stelle entstehen, welche viele der hier als notwendig erkannten Sensibilisierungs-, Weiterbildungs- und Koordinations- sowie Vernetzungsbemühungen übernehmen oder dafür Modellvorlagen erarbeiten könnte.

\section{Auswirkungen der Identifizierung}

28 Der formelle Identifizierungsentscheid hat in verschiedener Hinsicht Auswirkungen auf das Asylverfahren. Diese Auswirkungen bestehen auch dann, wenn kein Identifizierungsmodell wie das hier vorgeschlagene umgesetzt wird, sondern weiterhin bloss informelle Identifizierungen vorgenommen werden.

29 Für identifizierte Opfer muss eine Möglichkeit bestehen, ihren Aufenthalt entweder aus persönlichen Gründen oder zum Zweck der Kooperation mit den Strafverfolgungsbehörden zu regularisieren. Aktuell existieren in der Schweiz die folgenden Aufenthaltsoptionen für Menschenhandelsopfer: Eine Kurzaufenthaltsbewilligung für die Dauer des Strafverfahrens nach Art. 36 Abs. 2 VZAE, eine Härtefallbewilligung nach Art. 30 Abs. 1 lit. b AuG, die Asylgewährung nach Art. 49 AsylG sowie eine vorläufige Aufnahme nach Art. $83 \mathrm{AuG}$. Diese sind aber an unterschiedliche Voraussetzungen geknüpft und kommen somit nicht für alle Opfer gleichermassen in Frage. Sie schliessen sich zudem in den meisten Fällen gegenseitig aus: Wer ein Asylgesuch gestellt hat, ist gesetzlich von einer ausländerrechtlichen Bewilligung ausgeschlossen, und wer bereits den auslän-

35 Siehe die Darstellung der Identifizierungsmechanismen anderer Länder bei Frei, Menschenhandel und Asyl (Fn. 3), S. 451 ff. derrechtlichen Weg beschritten hat, dem kann eine verspätete und somit u.U. missbräuchliche Asylgesuchstellung vorgeworfen werden. Es ist auch nicht möglich, parallele Verfahren zu führen. Mit anderen Worten muss ein Menschenhandelsopfer sich sehr gut überlegen, auf welchem Weg es um eine Aufenthaltsbewilligung nachsucht, weil dieser Weg in der Regel nicht mehr verlassen werden kann. Eine Abhilfe dagegen könnte sein, dass im Rahmen der Identifizierungskommission oder - sofern keine solche besteht - des nationalen Weiterverweisungsmechanismus eine Kommunikation zwischen den Asylbehörden, den kantonalen Migrationsbehörden und der Rechtsvertretung der Opfer institutionalisiert wird, um die jeweils adäquate Aufenthaltsoption für ein Opfer zu bestimmen.

3o Wird nach dem Identifizierungsverfahren das davor suspendierte Asylverfahren weitergeführt oder neu eines eröffnet, so gilt prozessual eine (menschenrechtlich begründete) gesteigerte Untersuchungspflicht $3^{6}$ und das Dossier sollte an eine spezialisierte Person innerhalb des SEM übergeben werden, um das Verfahren durchzuführen. Der Identifizierungsentscheid ist bei der asylrechtlichen Sachverhaltserstellung zu berücksichtigen. Er hat aber keine automatischen Auswirkungen und es sollte verhindert werden, dass er eine negative Präjudizwirkung auf den Asylentscheid entfaltet. Auch eine Person, die nicht als Menschenhan-

$36 \overline{\text { So auch anerkannt vom Bundesverwaltungsge- }}$ richt in Urteil 2016/27 vom 18. Juli 2016, E. 10.2; darauf folgend erneut in den Urteilen D-2425/2017 vom 30. Mai 2017, S. 6 ff., D-5920/ 2016 vom 24. August 2017, E. 8.1. ff. und in Bezug auf sichere Drittstaaten Urteil E-1499/2016 vom 25. Januar 2017, E. 4.3.2. 
delsopfer anerkannt wurde, kann die Flüchtlingseigenschaft erfüllen oder Wegweisungsvollzugshindernisse aufweisen.

Erhält die Person kein Aufenthaltsrecht und muss sie die Schweiz verlassen, sind für identifizierte Opfer die Vorgaben für eine Rückkehr in Sicherheit und Würde zu beachten. Für die selbstorganisierte («freiwillige») Rückkehr besteht in der Schweiz ein positiv zu würdigendes Rückkehrhilfeprogramm für Opfer von Menschenhandel. Hingegen sind in Bezug auf die Anordnung und Ausgestaltung der Administrativhaft und den Vollzug einer Ausschaffung keine konkreten Vorgaben vorhanden; der Tatsache etwa, dass eine Inhaftierung oder zwangsweise Ausschaffung eines Opfers die Gefahr von Retraumatisierungen und die Anfälligkeit für re-trafficking erhöht, wird im schweizerischen Recht nicht Rechnung getragen. Es wäre deshalb notwendig, einheitliche Vorgaben sowohl auf nationaler als auch auf europäischer und/oder internationaler Ebene zu erarbeiten.

\section{Dublin-Verfahren}

32 Schliesslich stellt sich noch die Frage nach dem Opferschutz im DublinVerfahren. In der Praxis hat sich ein Grossteil37 der Menschenhandelsopfer bereits in einem anderen europäischen Staat aufgehalten und fällt somit häufig unter die Zuständigkeitskriterien der Dublin-III-Verordnung38. Diese enthält

37 Gemäss den oben zitierten Statistiken sind rund die Hälfte aller Verdachtsfälle auf Menschenhandel im Asylbereich im Dublin-Verfahren, siehe Frei, Menschenhandel und Asyl (Fn. 3), S. 61.

38 Verordnung (EU) Nr. 604/2013 des Europäischen Parlaments und des Rates vom 26. Juni 2013 zur Festlegung der Kriterien und Verfahren zur Bestimmung des Mitgliedstaats, der für die Prüfung keine expliziten Vorgaben zur Berücksichtigung der Situation von Menschenhandelsopfern. In der aktuellen Praxis der Schweiz wird dem Vollzug von Überstellungen im Dublin-System der Vorrang vor dem Opferschutz und (teilweise) sogar der Strafverfolgung gegeben. Aus völkerrechtlicher Sicht müssen die Opferrechte auch im Dublin-Verfahren gewährleistet werden, denn für den Opferschutz ist immer der aktuelle Aufenthaltsstaat des Opfers zuständig. Dies führt zu folgenden Ergebnissen für das Dublin-Verfahren:

- Da die Erholungs- und Bedenkzeit gemäss Art. 13 Europaratskonvention unabhängig vom Tatort zwingend zu gewähren ist und diese einen zuständigkeitsbegründenden Aufenthaltstitel i.S.v. Art. 19 Abs. 1 i.V.m. Art. 2 lit. 1 Dublin-III-Verordnung darstellt, erfolgt in der Regel ein automatischer Zuständigkeitsübergang auf die Schweiz, sobald bei einer Person konkrete Anhaltspunkte auf Menschenhandel erkannt werden. 39

- Für den Fall, dass der hier vertretenen Auslegung eines automatischen Zuständigkeitsübergangs nicht gefolgt wird, ist es vor dem Hintergrund der Verpflichtung zur internationalen Kooperation, der gesteigerten Untersuchungspflicht sowie im Hinblick auf das Vermeiden von re-trafficking notwendig, vor einer Überstellung zusammen mit dem zuständigen Staat zumindest den Empfang und die Be-

eines von einem Drittstaatsangehörigen oder Staatenlosen in einem Mitgliedstaat gestellten Antrags auf internationalen Schutz zuständig ist (DublinIII-Verordnung), ABl. L 180 vom 29. Juni 2013, S. 31.

39 Frei, Menschenhandel und Asyl (Fn. 3), S. 558 ff. 
treuung des Opfers zu klären. Im Sinne der Tarakhel-Rechtsprechung40 des EGMR müssten solche Garantien auch bei Menschenhandelsopfern eine Überstellungsvoraussetzung sein, was aber derzeit vom Bundesverwaltungsgericht abgelehnt wird. ${ }^{41}$

- Ist die Sicherheit des Opfers im zuständigen Staat fraglich, sollte ein Selbsteintritt gemäss Art. 17 Abs. 1 Dublin-III-Verordnung vorgenommen werden. Die teilweise angewendete Praxis des Bundesverwaltungsgerichts, die Sicherheit des zuständigen Staats basierend auf dessen Ratifikation der Europaratskonvention gegen Menschenhandel zu vermuten ${ }^{2}$, ist mit diesen Überlegungen, insbesondere angesichts der gesteigerten Abklärungspflicht bei Verdacht auf Menschenhandel, nicht vereinbar. 43

40 Urteil des EGMR [GC] 29217/12 vom 4. November 2014 (Tarakhel gegen Schweiz).

41 Urteile des Bundesverwaltungsgerichts D-1046/ 2017 vom 27. Februar 2017, S. 12 betreffend eine junge Frau aus Nigeria, die in Italien sexuell ausgebeutet worden ist; E-6874/2016 vom 12. Januar 2017, E. 5.5.1 betreffend eine junge Frau aus Kamerun, die vor ihrer Überfahrt nach Italien in Libyen Opfer sexueller Ausbeutung geworden ist.

42 Insbesondere in jüngeren Urteilen wird in textbausteinartiger Formulierung darauf hingewiesen, dass der zuständige Dublin-Staat die EKM ratifiziert habe und deshalb verpflichtet sei, Opfern von Menschenhandel eine angemessene Behandlung zu gewähren, so u.a. Urteile des Bundesverwaltungsgerichts E-4535/2017 vom 28. September 2017, E. 6.3; D-2690/2017 vom 18. Juli 2017, E. 5.4.1; E-6874/2016 vom 12. Januar 2017, E. 5.5.3; E-7919/2016 vom 29. Dezember 2016, S. 8; D-3362/2016 vom 10. Juni 2016, S. 14; E-4045/2016 vom 5. Juli 2016, E. 5.1; D-479/2016 vom 3. Februar 2016, S. 9; D-122/ 2016 vom 19. Januar 2016, S. 12; E-3515/2015 vom 17. Juni 2015 , S. 7.

43 Frei, Menschenhandel und Asyl (Fn. 3), S. 547 f.

\section{Handlungsbedarf}

33 Entsprechend der nach wie vor noch nicht sehr weit fortgeschrittenen Diskussion in der Schweiz um die Umsetzung der Opferschutzverpflichtungen im Asylverfahren, hat die Arbeit an vielen Stellen Handlungsbedarf identifiziert. Dieser lässt sich in fünf Dimensionen unterteilen: Verringerung der Relevanz des Tatbezugs zur Schweiz (1.), Verstärkung der Kooperation (2.), Fortführung und Ausbau der Sensibilisierungs- und Weiterbildungsmassnahmen (3.), Erarbeitung von Arbeitsinstrumenten (4.) sowie teilweise auch eine Notwendigkeit von Anpassungen de lege ferenda (5.).

\section{Relevanz des Tatbezugs zur Schweiz verringern}

34 Ein wesentliches Problem, das sich wie ein roter Faden durch die Arbeit zieht, ist der aktuell noch zu starke Fokus der Praxis auf den Tatbezug zur Schweiz und damit auf die «Nützlichkeit» der Opfer für die Strafverfolgungsbehörden, anstatt auf den Opferschutz als solchen. Dies zeigt sich etwa darin, dass im SEM zwar eine Federführung Menschenhandel existiert, diese aber als einziges «follow-up» bei konkreten Anhaltspunkten auf Menschenhandel eine Weiterleitung der Daten an das Bundesamt für Polizei (fedpol) vornimmt und keine Vernetzung mit spezialisierten Opferberatungsstellen erfolgt. Indem die Unterstützung für Menschenhandelsopfer in der Schweiz generell an die Opferhilfe geknüpft ist, haben zudem nur Opfer, die in der Schweiz ausgebeutet wurden, $\mathrm{Zu}$ gang zu dieser Unterstützung. Da auch die entsprechenden Abläufe für den Schutz der Opfer im Asylbereich nicht geklärt sind, ist in praktischer Hinsicht 
zu beobachten, dass dieser vor allem dann reibungslos verläuft, wenn eine Empfehlung der Strafverfolgungsbehörden vorliegt. Ist dies nicht der Fall, sind der Schutz und die Unterstützung der Opfer weitgehend abhängig vom Engagement einzelner Personen, z.B. der Rechtsvertretung, Opferberatungsstellen oder anderen Betreuungs- oder Vertrauenspersonen. 44 Dabei ist in der Regel ein erheblicher Aufwand dieser Personen notwendig, um eine Lösung im Sinne des Opferschutzes zu erreichen. Dass dies nicht nachhaltig ist, liegt auf der Hand.

Vielmehr sind Änderungen erforderlich, um den Schutz der Opfer im System selber zu verankern. Die nachfolgenden Massnahmengruppen zielen in diese Richtung.

\section{Kooperation}

36 Fast alle der in der Arbeit identifizierten Handlungsebenen machen in der einen oder anderen Weise auch eine Vernetzung und Kooperation zwischen den verschiedenen Akteuren des Opferschutzes notwendig. Dazu gehört u.a. die adäquate, koordinierte Reaktion auf ein «Verschwinden» eines potentiellen Opfers, eine Weiterverweisung der potentiellen Opfer an die Unterstützung gewährenden Stellen nach dem Erkennen konkreter Anhaltspunkte, eine Weiterverweisung in das Identifizierungsverfahren oder eine Verständigung zwischen den Migrationsbehörden und der Rechtsvertretung über die Aufenthaltsoptionen eines Opfers. Es sollte deshalb ein nationaler Weiterverweisungsmechanismus unter Einbezug aller beteiligten Stellen eingerichtet werden, in welchem alle Abläufe, Zuständig- keiten und auch Finanzierungsfragen geklärt werden. Beteiligt sein sollten alle Akteure, die in Kontakt mit potentiellen Opfern kommen könnten, d.h. die Asylbehörden selber, Betreuungspersonal, medizinisches Personal, die Rechtsvertretung, Vertrauenspersonen für unbegleitete Minderjährige, Sozialarbeitende oder Rechtsberatungsstellen; zudem auch die kantonalen Ausschaffungsgefängnisse und Rückkehrberatungsstellen und die relevanten Akteure des Opferschutzes ausserhalb des Asylbereichs, also die spezialisierten Opferberatungsstellen, Strafverfolgungs-, Migrations-, Sozial- und Kinderschutzbehörden sowie auch die für die Identifizierung zuständige Stelle. Darüber hinaus sollte auf nichtstaatlicher Ebene mindestens der Austausch und die Kooperation zwischen Rechtsberatungs- und Opferberatungsstellen institutionalisiert werden, dies auch im Hinblick auf die zentralere Rolle, die der Beratung und Rechtsvertretung im zukünftigen Asylverfahren ab 2019 zukommen werden. 45

\section{Sensibilisierung und Weiterbildung}

37 Eine flächendeckende, systematische Sensibilisierung aller Mitarbeitenden, die in Kontakt mit Menschenhandelsopfern kommen könnten, ist für die konkrete Umsetzung des Opferschutzes fundamental. Dazu gehören nebst den Sachbearbeiterinnen, Befragern und Entscheiderinnen im SEM auch die AsylbetreuerInnen, Medizinalpersonen, Sicherheitspersonal in den Unterkünften, Rechtsvertreterinnen und Rechtsberater, Vertrauenspersonen und gesetzliche Vertretungen von unbegleiteten Minderjährigen und Dolmet- 
schende. Da sich häufig auch erst nach Abschluss des Asylverfahrens Anzeichen auf Menschenhandel ergeben, müssen zusätzlich auch die Mitarbeitenden in Ausschaffungsgefängnissen, Rückkehrberatungsstellen sowie auf kantonalen Ämtern, wo die Ausreiseorganisation stattfindet, sensibilisiert werden. Gefordert ist dabei in erster Linie nicht eine "Ausbildung», sondern, wie erwähnt, eine Sensibilisierung. Es geht nicht darum, dass alle Mitarbeitenden dazu ausgebildet werden sollen, Opfer zu befragen oder eine abschliessende Einschätzung über die Opfereigenschaft zu treffen. Vielmehr ist das Ziel der Sensibilisierung, dass die Mitarbeitenden Anzeichen von Menschenhandel erkennen können, ein Verantwortungsbewusstsein entwickeln und wissen, welche Schritte bei einem Verdacht eingeleitet werden müssen. ${ }^{46}$

38 Zudem sind kontinuierliche Weiterbildungen für Mitarbeitende in Rechtsvertretung und -beratung sowie für die Sachbearbeitenden des SEM über die materiell- und verfahrensrechtlichen Besonderheiten bei Asylgesuchen von Menschenhandelsopfern notwendig.

\section{Arbeitsinstrumente}

39 Um den Opferschutz im konkreten Arbeitsalltag der Mitarbeitenden im Asylbereich umzusetzen, sollten Arbeitsinstrumente wie Leitfäden, Handbücher und dergleichen erarbeitet resp. überarbeitet und bereitgestellt werden. So könnte ein gesondertes Kapitel über die Beurteilung von Asylgesuchen von potentiellen Menschenhandelsopfern im Handbuch Asyl und Rückkehr47 des SEM geschaffen wer-

$46 \overline{\text { Frei, Menschenhandel und Asyl (Fn. 3), S. } 594 \text { f. }}$

47 SEM, Handbuch Asyl und Rückkehr. den. Auch die konkreten Vorgaben zur Durchführung eines Screenings an der Erstbefragung, die Definition des Vorgehens bei der Unterbringung von Menschenhandelsopfern, die Information der potentiellen Opfer über ihre Rechte und Pflichten, das Vorgehen im Falle eines Untertauchens von potentiellen (minderjährigen) Opfern, die Verwendung von asylspezifischen Indikatorenlisten oder gesamtschweizerische Standards für die Administrativhaft und die Ausreiseorganisation von Menschenhandelsopfern sollten schriftlich verankert werden. 48

\section{Legislative Anpassungen}

40 Einige der notwendigen Umsetzungsmassnahmen benötigen Gesetzes- oder Verordnungsänderungen.49 Konkret müssten aktuell für die Gewährung einer Erholungs- und Bedenkzeit durch das SEM (wenn dies nicht in den Aufgabenbereich der Identifizierungskommission fallen würde) sowie für die Pflicht zur Suspendierung des Asylverfahrens während des Identifizierungsverfahrens eine Rechtsgrundlage in der AsylV $1^{50}$ geschaffen werden. Die Suspendierung des Wegweisungsverfahrens durch die kantonalen Migrationsbehörden müsste in der VZAE verankert werden. Die Finanzierung der Unterstützung für Opfer im Asylverfahren müsste sich, je nach gewähltem Modell, auf eine Änderung des Anwendungsbereichs des OHG oder auf eine Grundlage in der AsylV 1 stützen können. Auch das vorgeschlagene Kommissionsmodell zur Identifizierung müsste in einem formellen Bundesgesetz verankert werden.

\footnotetext{
48 Frei, Menschenhandel und Asyl (Fn. 3), S. 595.

49 Frei, Menschenhandel und Asyl (Fn. 3), S. 595 f.

50 Asylverordnung 1 vom 11. August 1999 über Verfahrensfragen (AsylV 1; SR 142.311).
} 


\section{Ausblick}

41 Insgesamt lässt sich feststellen, dass der Asylbereich zum heutigen Zeitpunkt sowohl in Bezug auf die materielle Schutzgewährung als auch auf die Behandlung von Opfern im Verfahren sein Schutzpotential noch nicht vollständig ausschöpft. Die Arbeit versuchte, diese Lücken aufzuzeigen und Vorschläge zur Abhilfe zu entwickeln. Die Arbeit, die an vielen Stellen eine Neulandbegehung darstellt, hat auch zum Ziel, Grundlagen und Denkanstösse für eine vertiefte Diskussion über die Umsetzung der Opferschutzverpflichtungen in der Schweiz zu liefern. In dem Sinne bleibt zu hoffen, dass die Schlussfolgerungen und Vorschläge von der Wissenschaft und der Praxis aufgenommen und diskutiert werden. 